\title{
Algorithms for sensor chip alignment to blind datums
}

\author{
Gary B. Hughes \\ FLIR Systems Indigo Operations \\ 70 Castilian Drive \\ Goleta, California 93117 \\ E-mail: gary.hughes@ flir.com
}

\begin{abstract}
The sensor element of an imaging system should be mounted into its housing in such a way that the scene can be properly focused onto the sensor element's focal plane over the active area. Operational imaging requirements are forcing increasingly smaller tolerances on sensor alignment, and manufacturing systems must improve alignment capability to keep pace. Imaging system designs include reference datums that provide the basis for manufacturing alignment of optical components in each subassembly. Design constraints for alignment of the sensor element into the camera housing typically include $x, y, z$, clocking, and parallelism specifications. Measurement of $z$ and parallelism positioning is often problematic, since the relevant reference datum features are often beneath the mounting platform and are obscured to the measurement system. General algorithms for determining sensor chip alignment when datum features are inaccessible to the measurement system are described. Precharacterization measurements of datum surfaces are used to determine datum locations during alignment measurement. The algorithms are useful for active manufacturing alignment as well as postmounting alignment measurement. The algorithms are successfully implemented for ultraprecision, active manufacturing alignment, and postalignment measurement of IR imaging systems. () 2006 SPIE and IS\&T. [DOI: 10.1117/1.2234326]
\end{abstract}

\section{Introduction}

Alignment of the focal plane array (FPA) to the optical components is a critical design feature of an imaging system. In particular, optics with narrow depth of field characteristics require tight control over parallelism alignment of the FPA to the optical axis. It is not usually possible to correct for out-of-focus conditions with image processing techniques. Many applications, such as telescopes and satellite imaging systems, rely on alignment of optics to FPA during system integration (e.g., Refs. 1 and 2). Some systems, such as those containing multiple focal planes, are forced to pay more attention to alignment when the FPA is mounted to its platform. Some systems are produced in large quantities and alignment at system integration is not cost effective. For high-volume manufacturing, as for security/surveillance systems etc., statistical process control is utilized to track FPA alignment without measuring every FPA.

Measurement of FPA alignment at system integration is accomplished with test equipment and precision sources. ${ }^{3}$ This process can overcome minor misalignments of the FPA to its mounting platform. Minimizing the mounting error is still important, and even more so for systems that

Paper 05181RR received Oct. 7, 2005; revised manuscript received Feb. 15, 2006; accepted for publication Feb. 16, 2006; published online Jul. 24, 2006.

1017-9909/2006/15(3)/033003/12/\$22.00 @ 2006 SPIE and IS\&T. do not have the opportunity to align at system integration. In this case, accurate means are required for determining alignment of the FPA to its mounting platform based on measurements, for example, from optical/encoder systems of an aligner-bonder. Modern methods have been established to determine mechanical reference datums from optical measurements. ${ }^{4}$ A common problem in this context is determination of the datum locations on the mounting platform. Especially for parallelism alignment, the reference datum is often obscured from the aligner-bonder optics. This paper describes a process for determining the location of alignment datums that are obscured from measurement during FPA mounting.

\section{Background}

The sensor element (die) of an imaging system is typically mounted onto a platform using fiducial marks on the die and platform as reference points; a postmounting measurement is performed to verify die placement with respect to the reference datums. Design constraints for die alignment in the camera housing typically include $x, y, z$, clocking, and parallelism requirements (Fig. 1. These requirements often give rise to three orthogonal datum planes that are usually defined where specified surfaces on the platform contact mounting features in the camera housing. For the example presented here, Datum -A- is defined by three support pads (flat, coplanar surfaces) on the underside of a ceramic base. Datum -B- and Datum -C- are defined by points along edges of the ceramic base where the ceramic makes contact with a heat sink or other structural component. For the purpose of determining die alignment, these three datums are assumed to be exact planes, i.e., perfectly flat and mutually orthogonal. Ideally, the datums would be established by six points of contact: three on Datum -A-, two on Datum -B-, and one on Datum -C-.

The datum planes define a Euclidean coordinate system, and it is with respect to this datum coordinate system that die placement must be determined. The measurement coordinate system is typically defined by the axes of motion of the stages. The measurement coordinate system is (in general) different from the datum coordinate system of the part being aligned. An algorithm is presented that transforms measurements in the $z$-scope coordinate system to the datum coordinate system. Datum plane locations can be established within the $z$-scope coordinate system during die placement measurement or postmounting measurement.

Postmounting measurements are made by placing a sensor package into the measurement tool, and securing the 


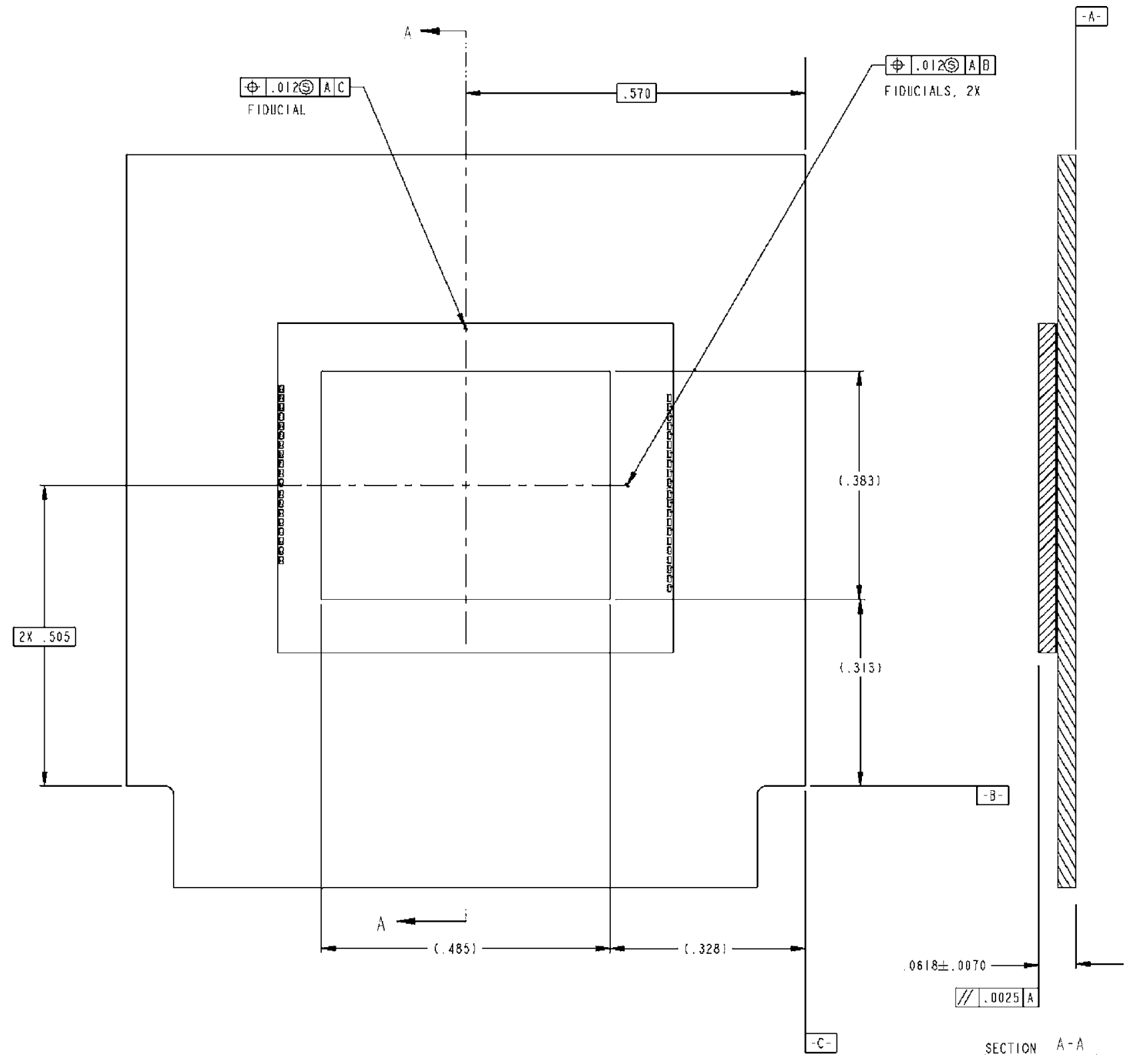

Fig. 1 Design specifications for sensor element (die) placement. Reference datums provide the basis for manufacturing alignment of the sensor element into the camera housing. Design constraints typically include $x, y, z$, clocking, and parallelism requirements. Here, the datums are three mutually orthogonal planes that create a Euclidean coordinate system (the "datum coordinate system"). Arbitrarily (and due to the layout of the datums), let Datum -A- be the $x-y$ plane; Datum -B- be the $x-z$ plane; and Datum -C- be the $y-z$ plane; $x$ alignment is constrained by stipulating the location of the top die fiducial to Datum -C-; $y$ alignment and clocking are constrained by stipulating the location of the two lateral die fiducials to Datum -B-, $z$ alignment to Datum -A- is stipulated at all three die fiducials. Since $z$ alignment is usually loose (optics can travel up and down), an additional parallelism requirement is stipulated to constrain the active area of the focal plane within the depth of focus of the optics; this requirement is more stringent than what is imposed by the tolerances in the $z$ alignment of the fiducials.

measurement tool on the $z$-scope stage. The measurement tool is designed to mimic the sensor package mounting contact points in the camera housing (heat sink). In particular, the contact points between the measurement tool and the sensor package's ceramic base are designed to be the same as the datum contact points between the ceramic base and the camera housing.

During postmounting measurement, the sensor package obscures Datum -A- from the z-scope optics, because this datum is on the underside of the ceramic base. To overcome this limitation, datum planes -A-, -B-, and -C- are determined by measuring fiducials that are permanently attached to the measurement tool. The relationship between the tool fiducials and datum planes is calculated from a precharacterization of the measurement tool. During precharacterization, repeated measurements of the tool fiducials and datum 
Table 1 The sequence of measurements and calculations used for tool precharacterization.

Measure tool fiducial points, and Datum -A-, Datum -B-, and Datum -C- points on the bare measurement tool

Calculate Datum -A- "best-fit" plane equation

Calculate average tool fiducial locations

Calculate tool fiducial plane equation

Calculate the first three plane transfer values, the distances along the tool fiducial plane normal vector to the virtual points $F A_{1}, F A_{2}$, $F A_{3}$ in the Datum -A- plane

"Level" the three virtual points $F A_{1}, F A_{2}$, and $F A_{3}$ and Datum -B- points to the Datum -A- plane

Calculate "best-fit" line for "leveled" Datum -B- points

Calculate Datum -B- plane equation from line slope and intercept parameters

Calculate vector perpendicular to $\left\langle F A_{3}^{\prime}-F A_{1}^{\prime}\right\rangle$ in the Datum -A- plane

Calculate the next two plane transfer values, the distances along the, $F A_{-} B$ vector to the two virtual points $F B_{1}$ and $F B_{2}$ in the

Datum -B- plane

"Level" Datum -C- points to the

Datum -A- plane

"Skew" the three ("leveled") virtual points

$F A_{1}^{\prime}, F A_{2}^{\prime}$ and $F A_{3}^{\prime}$ and the "leveled"

Datum -C- points to the Datum -B- plane

Calculate Datum -C- plane equation

Calculate vector perpendicular to

$\left\langle F A_{2}^{\prime}-F A_{1}^{\prime}\right\rangle$ in the Datum -A- plane

Calculate the final plane transfer value, the distance along the $F A_{-} C$ vector to the virtual point $F C_{1}$ in the Datum -C- plane
$\left\{F:\left(x_{i}, y_{i}, z_{i}\right), i=1 \ldots n_{F}\right\}$ for each fiducial

$\left\{-P_{-}:\left(x_{i}, y_{i}, z_{i}\right), i=1 \ldots n_{-P_{-}}\right\}$for each datum

$$
N_{-A-} \cdot\langle x, y, z\rangle=d_{-A-}
$$

(1) and (2)

(3a) and (3b)

$$
\begin{gathered}
\bar{F}_{1}, \bar{F}_{2}, \bar{F}_{3} \\
N_{-F-} \cdot\langle x, y, z\rangle=d_{-F-} \\
t_{1}=\frac{d_{-A-}-N_{-A-} \cdot \bar{F}_{1}}{N_{-A-} \cdot N_{-F-}}, t_{2}=\frac{d_{-A-}-N_{-A_{-}} \cdot \bar{F}_{2}}{N_{-A_{-}} \cdot N_{-F-}} \\
t_{3}=\frac{d_{-A_{-}-}-N_{-A-} \cdot \bar{F}_{3}}{N_{-A-} \cdot N_{-F-}} \\
F A_{i}^{\prime}=\left[R_{\langle x, y, z\rangle}\right] \cdot F A_{i}, i=1 \ldots 3 \\
B_{i}^{\prime}=\left[R_{\langle x, y, z\rangle}\right] \cdot B_{i}, i=1 \ldots n_{-B-} \\
y=m x+b \\
N_{-B-} \cdot\langle x, y, z\rangle=d_{-B-}
\end{gathered}
$$

$$
F A_{-} B
$$

$$
\begin{gathered}
t_{4}=\frac{d_{-B_{-}}-N_{-B_{-}} \cdot F A_{1}^{\prime}}{N_{-B_{-}} \cdot F A_{-} B} \\
t_{5}=\frac{d_{-B_{-}}-N_{-B_{-}} \cdot F A_{3}^{\prime}}{N_{-B_{-}} \cdot F A_{-} B} \\
C_{i}^{\prime}=\left[R_{\langle x, y, z\rangle}\right] \cdot C_{i}, i=1 \ldots n_{-C-} \\
F A_{i}^{\prime \prime}=\left[R_{\langle x, y\rangle}\right] \cdot F A_{i}^{\prime}, i=1 \ldots 3 \\
C_{i}^{\prime \prime}=\left[R_{\langle x, y\rangle}\right] \cdot C_{i}^{\prime}, i=1 \ldots n_{-C-} \\
N_{-C-} \cdot\langle x, y, z\rangle=d_{-C-} \\
F A_{-} C
\end{gathered}
$$

$$
t_{6}=\frac{d_{-C_{-}-}-N_{-C_{-}} \cdot F A_{1}^{\prime \prime}}{N_{-C_{-}} \cdot F A_{-} C}
$$

Note: The "plane transfer values" of Eqs. (10), (20), and (27) capture the fixed relationship between the measurement tool fiducials and the datum planes. These values can be used to establish the location of the datum planes from measurement of the tool fiducials alone, even if the datum planes are obscured to the measurement system.

surfaces are made without a sensor package loaded in the tool, so all datum surfaces are visible to the $z$-scope measurement system. The fiducial-datum relationships are calculated as "plane transfer values," which are scalars that denote the distance from the fiducials to the datum planes along vectors determined by the fiducial locations. There are six plane transfer values: three for Datum -A-, two for Datum -B-, and one for Datum -C-.

The mathematical algorithms presented in this paper are not novel; however, the combination of steps presented can be useful for determining blind datum locations. All the steps are shown so that the output of one step leads to the input of another. The algorithms are explicitly stated so that their compatibility is clear, and to make them all fit together to accomplish the alignment.

Tool fiducials and precharacterization measurements are used to determine datum locations during the postmounting die alignment measurement routine. The set of precharacterization measurements must be sufficient to determine the locations of all three datum planes from measurements of the three tool fiducials. The idea here is that the spatial relationship between the fiducials and datums does not change when a sensor package is placed in the tool, or when the orientation of the tool changes. The fixed relation- 


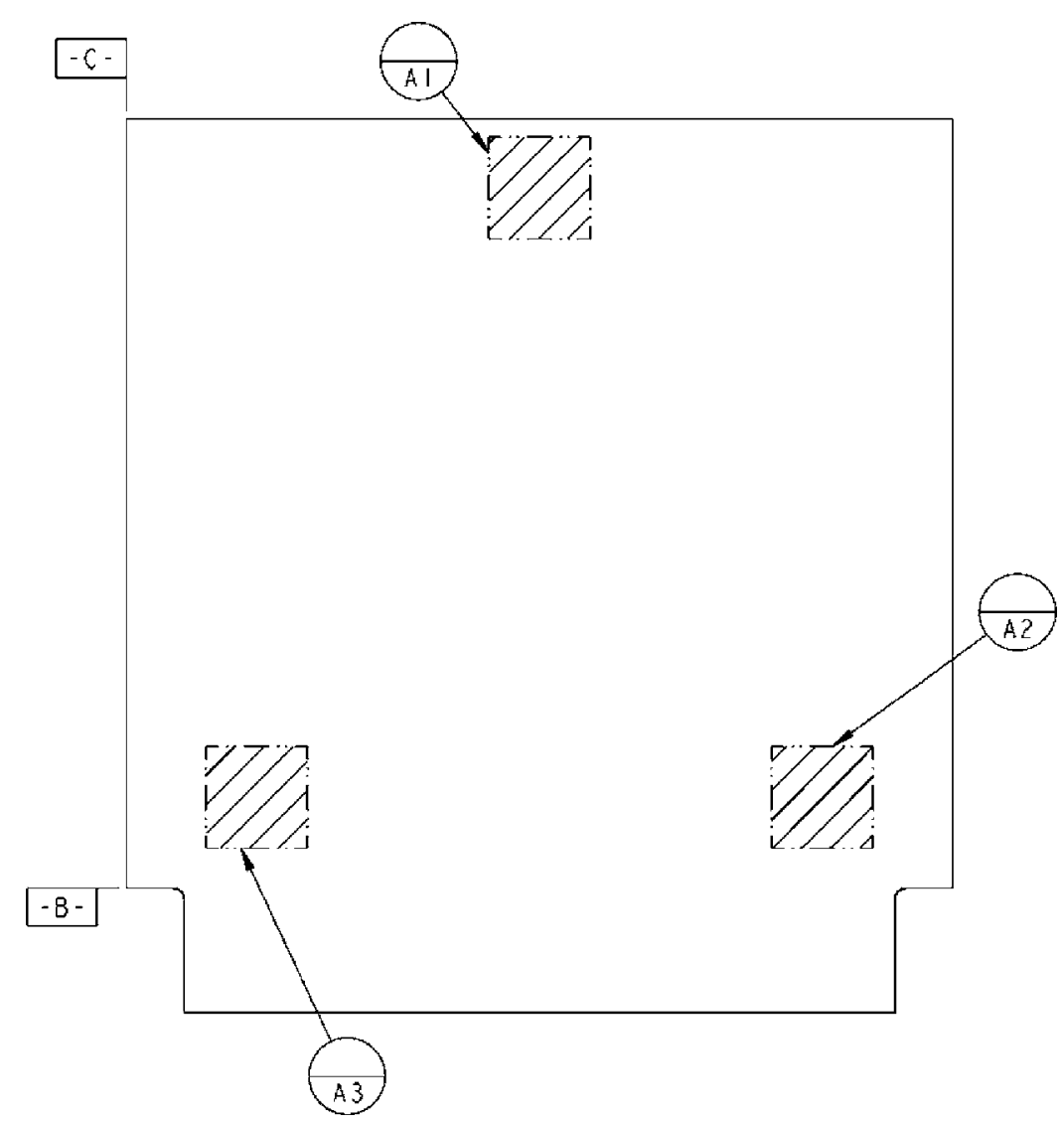

Fig. 2 Three datum planes are established by surfaces on the ceramic base. For the purpose of determining die alignment, these three datums are assumed to be exact planes, i.e., perfectly flat and mutually orthogonal. Ideally, the datums would be established by six points of contact: three on Datum -A-, two on Datum -B-, and one on Datum -C-.

ship between fiducials and datums can be determined by precharacterization measurements; the fixed relationships can then be exploited to determine datum locations from fiducial measurements during postmounting measurement of die placement. This approach is essential for a $z$-scope measurement system, since Datum -A- cannot be viewed with $z$-scope optics once a sensor package is placed in the measurement tool. The sequence of measurements and calculations in Table 1 provide a method for establishing the relationship between fiducials and datums in the measurement tool. Table 2 gives a sequence of measurements and calculations for using the fixed relationship to establish the location of the three datum planes within the $z$-scope measurement system.

\section{Tool Precharacterization}

Three fiducials designated $F_{1}, F_{2}$, and $F_{3}$ are mounted on the measurement tool in such a way that they can be viewed by the measurement system during alignment or postalignment measurement. Additionally, in the context of the algorithms presented in this paper, $F_{1}$ is near the datum origin on the measurement tool, $F_{2}$ is out the positive $y$ axis of the measurement system, and $F_{3}$ is out the positive $x$ axis of the measurement system. These designations are arbitrary, but some steps in the sequence of calculations depend on this configuration. The basis of the method presented here is that the spatial relationship between the tool fiducials and the datum surfaces on the tool does not change when a sensor package to be measured is placed in the tool, or when the orientation of the tool changes within the measurement coordinate system of the $z$ scope. The fixed relationship between fiducials and datums can be determined by precharacterization measurements and datum transfer parameters are calculated from the measurements. The datum transfer parameters are stored, and used later to determine datum locations from fiducial measurements during active alignment or postmounting measurement of die placement.

To establish the relationship between fiducials and datums, repeated measurements of the fiducials and datum surfaces are made without a sensor package mounted in the tool. Repeating the measurements allows random errors to be averaged out. For example,

$\begin{array}{cl}6 \times\{X \text { Y Z F1 } & \text { Tool Fiducial } F_{1} \\ \text { XY Z F2 } & \text { Tool Fiducial } F_{2} \\ \text { X Y Z F3 } & \text { Tool Fiducial } F_{3} \\ 5 \times\{X Y Z \text { A1 }\} & \text { Five points on Pad } 1 \text { of Datum -A- surface } \\ 5 \times\{X \text { Y Z A2 }\} & \text { Five points on Pad } 2 \text { of Datum -A- surface } \\ 5 \times\{X \text { Y Z A3 }\} & \text { Five points on Pad } 3 \text { of Datum -A- surface } \\ \} & \\ 6 \times\{X Y Z \text { F1 } & \text { Tool Fiducial } F_{1}\end{array}$




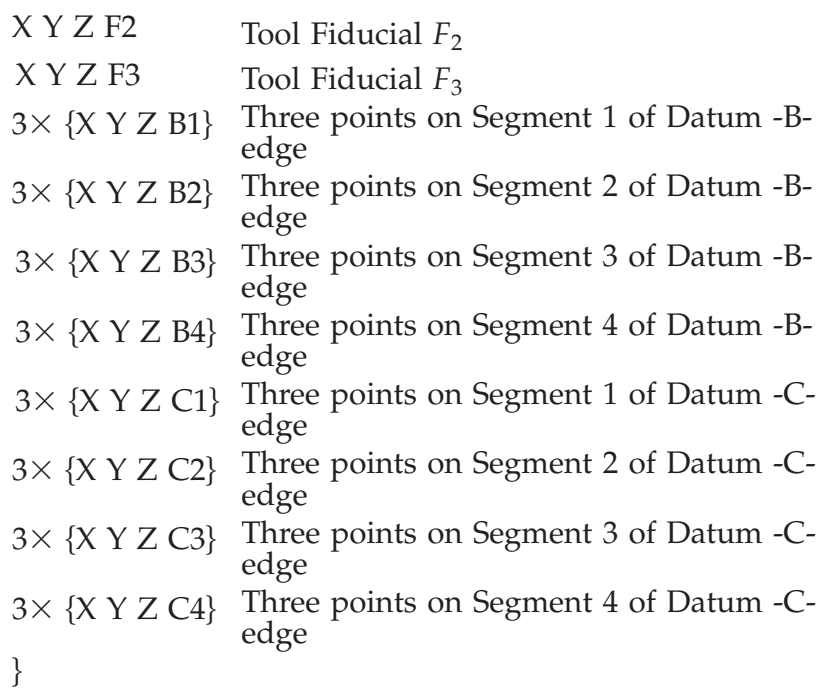

This series of measurements would produce the following data sets for each relevant feature:

$$
\begin{aligned}
& \left\{F_{1}:\left(x_{i}, y_{i}, z_{i}\right), i=1 \ldots n_{F_{1}}\right\} \\
& \left\{F_{2}:\left(x_{i}, y_{i}, z_{i}\right), i=1 \ldots n_{F_{2}}\right\} \\
& \left\{F_{3}:\left(x_{i}, y_{i}, z_{i}\right), i=1 \ldots n_{F_{3}}\right\} \\
& \left\{A:\left(x_{i}, y_{i}, z_{i}\right), i=1 \ldots n_{A}\right\} \\
& \left\{B:\left(x_{i}, y_{i}, z_{i}\right), i=1 \ldots n_{B}\right\} \\
& \left\{C:\left(x_{i}, y_{i}, z_{i}\right), i=1 \ldots n_{C}\right\}
\end{aligned}
$$

Datum -A- is a plane defined by the three support pads (Fig. 2). Standard methods can be used to establish the location of the Datum -A- plane in the measurement coordinate system. The plane equation for Datum -A- is determined from the measured points by linear least-squares. For a set of data values $\left\{A:\left(x_{i}, y_{i}, z_{i}\right), i=1 \ldots n_{A}\right\}$, the linear least-squares solution for "best-fit" plane equation can be found by solving the linear system (e.g., Ref. 5)

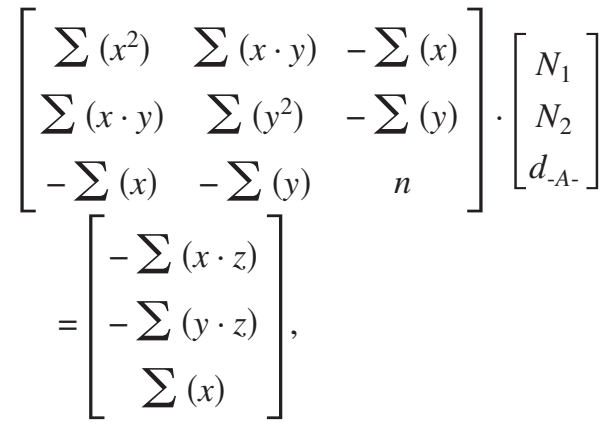

where the "best-fit" plane normal vector is given by $N_{-A}$ $=\left\langle N_{1}, N_{2}, 1\right\rangle$, and the "best-fit" plane equation for Datum -A- is
$N_{1} \cdot x+N_{2} \cdot y+z=d_{-A-}$

The values of $N_{1}, N_{2}$, and $d_{-A \text { - }}$ can be found by solving the linear system, for example by using Gaussian elimination with pivot scaling and back substitution (e.g., Ref. 6). Since the Datum -A- surface is predominantly level and flat in the context of the $z$-scope measurement system, the linear system is not expected to be ill-conditioned, and some shortcuts to solving the system may be employed safely. For the shortcut solution described here, the $3 \times 3$ linear system can be modified by translating the data to cluster around the origin:

$$
\begin{gathered}
{\left[\begin{array}{ccc}
\sum(x-\bar{x})^{2} & \sum(x-\bar{x}) \cdot(y-\bar{y}) & -\sum(x-\bar{x}) \\
\sum(x-\bar{x}) \cdot(y-\bar{y}) & \sum(y-\bar{y})^{2} & -\sum(y-\bar{y}) \\
-\sum(x-\bar{x}) & -\sum(y-\bar{y}) & n
\end{array}\right]} \\
\cdot\left[\begin{array}{c}
N_{1} \\
N_{2} \\
d_{-A-}
\end{array}\right]=\left[\begin{array}{c}
-\sum(x-\bar{x}) \cdot(z-\bar{z}) \\
-\sum(y-\bar{y}) \cdot(z-\bar{z}) \\
\sum(x-\bar{x})
\end{array}\right]
\end{gathered}
$$

Since the sums $\Sigma(x-\bar{x})$ and $\Sigma(y-\bar{y})$ should evaluate to zero, the $3 \times 3$ system is simplified to a $2 \times 2$ system:

$$
\begin{aligned}
& {\left[\begin{array}{ccc}
\sum(x-\bar{x})^{2} & \sum(x-\bar{x}) \cdot(y-\bar{y}) & 0 \\
\sum(x-\bar{x}) \cdot(y-\bar{y}) & \sum(y-\bar{y})^{2} & 0 \\
0 & 0 & n
\end{array}\right] \cdot\left[\begin{array}{l}
N_{1} \\
N_{2} \\
d_{-A-}
\end{array}\right]} \\
& =\left[\begin{array}{c}
-\sum(x-\bar{x}) \cdot(z-\bar{z}) \\
-\sum(y-\bar{y}) \cdot(z-\bar{z}) \\
0
\end{array}\right] \\
& \Rightarrow\left[\begin{array}{cc}
\sum(x-\bar{x})^{2} & \sum(x-\bar{x}) \cdot(y-\bar{y}) \\
\sum(x-\bar{x}) \cdot(y-\bar{y}) & \sum(y-\bar{y})^{2}
\end{array}\right] \cdot\left[\begin{array}{l}
N_{1} \\
N_{2}
\end{array}\right] \\
& =\left[\begin{array}{l}
-\sum(x-\bar{x}) \cdot(z-\bar{z}) \\
-\sum(y-\bar{y}) \cdot(z-\bar{z})
\end{array}\right] \text {. }
\end{aligned}
$$

Algebraic solution of the $2 \times 2$ system can be computed directly:

denom $=\sum(x-\bar{x})^{2} \cdot \sum(y-\bar{y})^{2}-\left[\sum(x-\bar{x}) \cdot(y-\bar{y})\right]^{2}$, 
Table 2 The sequence of measurements and calculations used for operational determination of datum locations from measurements of the tool fiducial locations.

Measure tool fiducial points once each

Calculate tool fiducial plane equation

Using the first three plane transfer values, calculate the location of the virtual points

$F A_{1}, F A_{2}$, and $F A_{3}$ in the Datum -A- plane

Calculate Datum -A- plane equation from the virtual points $F A_{1}, F A_{2}$ and $F A_{3}$

Calculate vector perpendicular to $\left\langle F A_{3}-F A_{1}\right\rangle$ in the Datum -A- plane

Using the next two plane transfer values, the calculate the location of the virtual points $F B_{1}$ and $F B_{2}$ in the Datum -B- Plane

Calculate Datum -B- plane equation from the virtual points $F B_{1}$ and $F B_{2}$

Calculate vector perpendicular to $\left\langle F A_{2}-F A_{1}^{\prime}\right\rangle$ in the Datum -A- plane

Using the final plane transfer value, calculate the location of the virtual point $F C_{1}$ in the Datum -C- Plane

Calculate Datum -C- plane equation from the virtual point $F C_{1}$

Determine the Datum Origin, the intersection of the three datum planes by solving the three plane equations simultaneously

$$
\begin{gathered}
\left\{F_{i}:(x, y, z), i=1 \ldots 3\right\} \\
N_{-F_{-}} \cdot\langle x, y, z\rangle=d_{-F-} \\
F A_{1}=\begin{array}{l}
F_{1}+t_{1} * N_{-F-}, F A_{2}=F_{2}+t_{2} * N_{-F-} \\
F A_{3}=F_{3}+t_{3} * N_{-F-}
\end{array} \\
N_{-A_{-} \cdot\langle x, y, z\rangle=d_{-A_{-}}} \text {(4) and (5) } \\
F A_{-} B \\
F B_{1}=F A_{1}+t_{4} * F A_{-} B F B_{2}=F A_{2}+t_{5} * F A_{-} B \\
N_{-B-} \cdot\langle x, y, z\rangle=d_{-B-} \\
F A_{-} C \\
F C_{1}=F A_{1}+t_{6} * F A_{-} C
\end{gathered}
$$

Datum Origin (1) Datum Origin (2) Datum Origin (3)

The six "plane transfer values" established from precharacterization measurements (Table 1) are required. Measurements of the tool fiducials are made, and the datum surface locations are calculated by the sequence above. No direct measurements of the datum surfaces are required to establish their locations.

$\left[\begin{array}{l}N_{1} \\ N_{2}\end{array}\right]=\left[\begin{array}{c}\frac{\sum(x-\bar{x}) \cdot(y-\bar{y}) \cdot \sum(y-\bar{y}) \cdot(z-\bar{z})-\sum(y-\bar{y})^{2} \cdot \sum(x-\bar{x}) \cdot(z-\bar{z})}{\operatorname{denom}} \\ \frac{\sum(x-\bar{x}) \cdot(y-\bar{y}) \cdot \sum(x-\bar{x}) \cdot(z-\bar{z})-\sum(x-\bar{x})^{2} \cdot \sum(y-\bar{y}) \cdot(z-\bar{z})}{\text { denom }}\end{array}\right]$.

A denominator close to zero would indicate that the linear system is close to being singular. However, if all the measurements are valid, the singular case should not occur. The complete "best-fit" plane equation is established from the normal vector by requiring the plane to pass through the data centroid:

$\left\langle N_{1}, N_{2}, 1\right\rangle \cdot\langle x, y, z\rangle=\left\langle N_{1}, N_{2}, 1\right\rangle \cdot\langle\bar{x}, \bar{y}, \bar{z}\rangle$

By default (the way the linear system is set up), the normal vector is pointing upward. For convenience, the plane nor- mal vector can be scaled to unit length, and the Datum -Aplane equation becomes:

$N_{-A^{-}} \cdot\langle x, y, z\rangle=d_{-A_{-}}$,

where

$$
N_{-A-}=\frac{\left\langle N_{1}, N_{2}, 1\right\rangle}{\left\|\left\langle N_{1}, N_{2}, 1\right\rangle\right\|_{2}} \text { and } d_{-A-}=\frac{\left\langle N_{1}, N_{2}, 1\right\rangle \cdot\langle\bar{x}, \bar{y}, \bar{z}\rangle}{\left\|\left\langle N_{1}, N_{2}, 1\right\rangle\right\|_{2}} \text {. }
$$

Once the Datum -A- plane equation is known, the next 


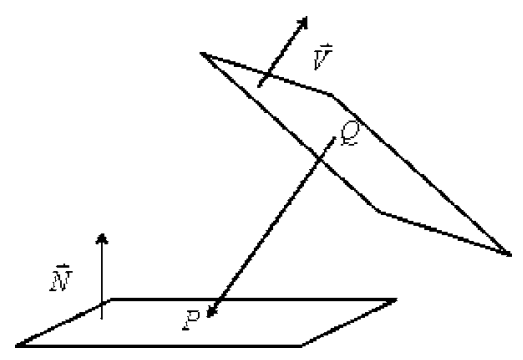

Fig. 3 A "plane transfer value" is the distance from a point $Q$ in the first plane to a point $P$ in the second plane, along the normal vector of the first plane.

step is to find the tool fiducial plane equation. The fiducial plane equation is determined from direct measurements of the three fiducials.

$\left\{F_{1}:\left(x_{i}, y_{i}, z_{i}\right), i=1 \ldots n_{F_{1}}\right\}$

$\left\{F_{2}:\left(x_{i}, y_{i}, z_{i}\right), i=1 \ldots n_{F_{2}}\right\}$

$\left\{F_{3}:\left(x_{i}, y_{i}, z_{i}\right), i=1 \ldots n_{F_{3}}\right\}$.

Since multiple measurements of each fiducial are available, the best approach is to use the mean measured locations for all calculations. For three average fiducial locations $\bar{F}_{1}, \bar{F}_{2}$, and $\bar{F}_{3}$, the normal vector (unit length, and pointing upward) can be determined using cross products (e.g., Ref. 7):

$$
\begin{aligned}
N_{-F-}= & \left\langle\left\langle\bar{F}_{2}-\bar{F}_{1}\right\rangle\right. \\
& \left.\times\left\langle\bar{F}_{3}-\bar{F}_{1}\right\rangle\right\rangle \cdot \frac{\operatorname{sign}\left\lfloor\left\langle\left\langle\bar{F}_{2}-\bar{F}_{1}\right\rangle \times\left\langle\bar{F}_{3}-\bar{F}_{1}\right\rangle\right\rangle(3)\right\rfloor}{\left\|\left\langle\bar{F}_{2}-\bar{F}_{1}\right\rangle \times\left\langle\bar{F}_{3}-\bar{F}_{1}\right\rangle\right\|_{2}},
\end{aligned}
$$

$N_{-F-}$ is the fiducial plane normal vector (unit length, and pointing upward), and the fiducial plane equation is

$N_{-F-} \cdot\langle x, y, z\rangle=N_{-F-} \cdot \bar{F}_{1}$,

where the right-hand side of the equation is the plane $d$ value $\left(d_{-F_{-}}=N_{-F_{-}} \cdot F_{1}\right)$.

Two plane equations (fiducial plane, datum plane) are now known, calculated from the precharacterization data per Eqs. (1), (2), (3a), (3b), (4), and (5). The main assumption for the method presented in this paper is that the relationship between these two planes is fixed, regardless of the orientation of the tool, or the presence of a sensor package mounted in the tool. The relationship between the two planes can be described mathematically by three "plane transfer values" representing the distance from the fiducial points to the Datum -A- plane along the fiducial plane normal vector. This general approach is depicted in Fig. 3. The "plane transfer values" are then stored for later use when the datum surface is obscured to the measurement system; measurements of the fiducials can be used with the "plane transfer values" to calculate the datum location.

Textbooks often give a formula for the shortest distance from a given point to a known plane (e.g., Ref. 7). The shortest distance from the point to the plane is along the

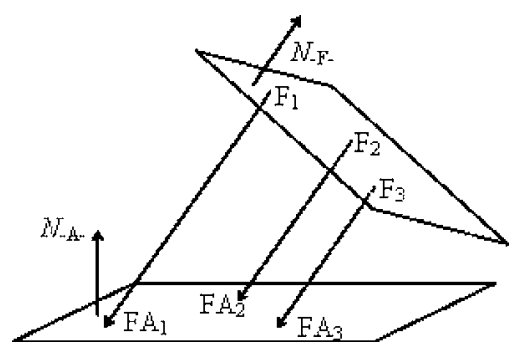

Fig. 4 "Plane transfer values" from the tool fiducial locations to the Datum -A- plane, along the normal vector of the fiducial plane $\left(N_{-F_{-}}\right)$.

plane's normal vector to the destination in the plane. In the current application, the destination plane will be unknown (since Datum -A- cannot be measured during alignment); in particular, the plane's normal vector will not be available. A formula is required for the distance from a given point to a known plane along an arbitrary vector, which is a slight modification to the usual textbook formula. The new objective is to arrive at a point $P$ in a plane with plane equation $\vec{N} \cdot\langle x, y, z\rangle=d_{N}$, starting from a point $Q$ in a plane with plane equation $\vec{V} \cdot\langle x, y, z\rangle=d_{V}$. The vector from $Q$ to $P$ will, for convenience, be in the direction of the normal vector $V$ (Fig. 3). This is convenient because the upper plane will be known in the current application (from fiducial measurements). Starting with $\vec{N} \cdot\langle x, y, z\rangle=d_{N}$, if the point $P$ is in this plane, then from the definition of a plane, the following relation holds (as for any point $P$ in the plane):

$N \cdot P=d_{N}$

The point $P$ is a certain distance $t$ away from the point $Q$ along the vector $\vec{V}$ :

$P=Q+t \cdot \vec{V}$

Substituting the alternate expression for $P[\mathrm{Eq} .(7)]$ into the lower plane Eq. (6) gives

$\vec{N} \cdot(Q+t \cdot \vec{V})=d_{N}$.

Solving algebraically for the distance $t$ in Eq. (8) gives the "plane transfer value:"

$t=\frac{d_{N}-\vec{N} \cdot Q}{\vec{N} \cdot \vec{V}}$.

As depicted in Fig. 4, three plane transfer values are calculated from the Datum -A-plane Eq. (3a) and (3b) and the fiducial plane Eq. (5), based on data collected during tool characterization: 
$t_{1}=\frac{d_{-A^{-}}-N_{-A^{-}} \cdot F_{1}}{N_{-A^{-}} \cdot N_{-F-}}=( \pm)\left\|F_{1}-F A_{1}\right\|_{2}$,

$t_{2}=\frac{d_{-A_{-}}-N_{-A^{-}} \cdot F_{2}}{N_{-A^{-}} \cdot N_{-F-}}=( \pm)\left\|F_{2}-F A_{2}\right\|_{2}$,

$t_{3}=\frac{d_{-A-}-N_{-A-} \cdot F_{3}}{N_{-A-} \cdot N_{-F-}}=( \pm)\left\|F_{3}-F A_{3}\right\|_{2}$.

The plane transfer values have a magnitude that is equal to the distance between each of the three points and their corresponding "nonorthogonal projections" onto the Datum -A-plane, and a sign that depends on the direction of the fiducial plane normal vector.

The "plane transfer values," along with measurements of the tool fiducials, can be used during operational alignment to establish the datum location. The three virtual points $F A_{1}, F A_{2}$, and $F A_{3}$ in the Datum -A- plane (Fig. 4) are calculated from the measured values of $F_{1}, F_{2}$, and $F_{3}$, and the fiducial plane equation by using the plane transfer values, and the normal vector to the fiducial plane:

$$
\begin{gathered}
F A_{1}=F_{1}+t_{1} * N_{-F-}, \quad F A_{2}=F_{2}+t_{2} * N_{-F-}, \\
F A_{3}=F_{3}+t_{3} * N_{-F-} .
\end{gathered}
$$

The Datum -A- plane equation can be calculated from the three virtual points by

$$
\begin{aligned}
N_{-A-}= & \left\langle\left\langle F A_{2}-F A_{1}\right\rangle \times\left\langle F A_{3}-F A_{1}\right\rangle\right\rangle \\
& \cdot \frac{\operatorname{sign}\left[\left\langle\left\langle F A_{2}-F A_{1}\right\rangle \times\left\langle F A_{3}-F A_{1}\right\rangle\right\rangle(3)\right]}{\left\|\left\langle F A_{2}-F A_{1}\right\rangle \times\left\langle F A_{3}-F A_{1}\right\rangle\right\|_{2}} .
\end{aligned}
$$

Note that $N_{-A_{-}}$is the Datum -A-plane normal vector (unit length, and pointing upward), and the plane equation is

$N_{-A_{-}} \cdot\langle x, y, z\rangle=N_{-A_{-}} \cdot F A_{1}$

where the right-hand side of the equation is the plane $d$ value $\left(d_{-A_{-}}=N_{-A_{-}} \cdot F A_{1}\right)$. As a check of the "plane transfer value" method, the two equations for the Datum -A-plane [Eqs. (3a), (3b), and (13)] should be identical.
The three virtual points $F A_{1}, F A_{2}$, and $F A_{3}$ in Datum -A-, along with additional precharacterization measurements, can also be used to determine the two other mutually orthogonal datum planes (Datums -B- and -C-). To establish the location of Datum -B-, the plane normal vector is required. The Datum -B- normal vector is orthogonal to the Datum -A- normal vector (by definition). By finding a vector that lies within Datum -B- (parallel to Datum -B-), the Datum -B- normal vector can be calculated by crossproduct with the Datum -A- normal vector. A vector lying within the Datum -B- plane can be found from the measured points. During tool precharacterization, several points are measured on the edges of the tool that represent Datum -B- and Datum -C-:

$\left\{B:\left(x_{i}, y_{i}, z_{i}\right), i=1 \ldots n_{B}\right\}, \quad\left\{C:\left(x_{i}, y_{i}, z_{i}\right), i=1 \ldots n_{C}\right\}$.

To simplify the remaining calculations, the three virtual points $F A_{1}, F A_{2}$, and $F A_{3}$, and all of the measured points on Datums -B- and -C-, can be "leveled" to the Datum -Aplane (equivalent to "projecting" the points orthogonally onto the Datum -A- Plane). When this is done, the $z$ values for each of the measured points along Datums -B- and -Cbecome irrelevant. A rotation axis (and a rotation angle) for "leveling" points to Datum -A- can be found by cross product (and dot product) between the Datum -A- plane normal vector and the $z$ axis (e.g., Ref. 7):

$$
\begin{aligned}
& R_{\langle x, y, z\rangle}= \frac{\left\langle N_{-A_{-}} \times\langle 0,0,1\rangle\right\rangle}{\left\|N_{-A_{-}} \times\langle 0,0,1\rangle\right\|_{2}} \\
& {[\text { The rotation axis for "leveling" to }} \\
&-\mathrm{A}-, \text { a (unit length) vector }], \\
& \theta=\arccos \left[\frac{N_{-A_{-}} \cdot\langle 0,0,1\rangle}{\left\|N_{-A}\right\|_{2}}\right]
\end{aligned}
$$

[The rotation angle for "leveling" to

$$
\text { - A - , a scalar]. }
$$

The rotation matrix corresponding to the rotation axis and rotation angle calculated by Eqs. (14a) and (14b), respectively, is

$$
\left[R_{\langle x, y, z\rangle}\right]=\left[\begin{array}{ccc}
\cos \theta+x^{2} \cdot(1-\cos \theta) & -z \cdot \sin \theta+x \cdot y \cdot(1-\cos \theta) & y \cdot \sin \theta+x \cdot z \cdot(1-\cos \theta) \\
z \cdot \sin \theta+x \cdot y \cdot(1-\cos \theta) & \cos \theta+y^{2} \cdot(1-\cos \theta) & -x \cdot \sin \theta+y \cdot z \cdot(1-\cos \theta) \\
-y \cdot \sin \theta+x \cdot z \cdot(1-\cos \theta) & x \cdot \sin \theta+y \cdot z \cdot(1-\cos \theta) & \cos \theta+z^{2} \cdot(1-\cos \theta)
\end{array}\right]
$$

The rotation matrix is calculated, then applied to the three virtual points $F A_{1}, F A_{2}$, and $F A_{3}$, and all of the measured points on Datums -B- and -C-, by left-multiplication to generate a set of "leveled" points (Fig. 5):

$F A_{i}^{\prime}=\left[R_{\langle x, y, z\rangle}\right] \cdot F A_{i}^{\prime}, i=1 \ldots 3$,

$$
\begin{aligned}
& B_{i}^{\prime}=\left[R_{\langle x, y, z\rangle}\right] \cdot B_{i}^{\prime}, i=1 \ldots n_{-B-}, \\
& C_{i}^{\prime}=\left[R_{\langle x, y, z\rangle}\right] \cdot C_{i}^{\prime}, i=1 \ldots n_{-C-} .
\end{aligned}
$$

When the three virtual points $F A_{1}, F A_{2}$, and $F A_{3}$, and all of the measured points on Datums -B- and -C-, have been "leveled" to Datum -A-, the resulting $(x, y)$ values can be 


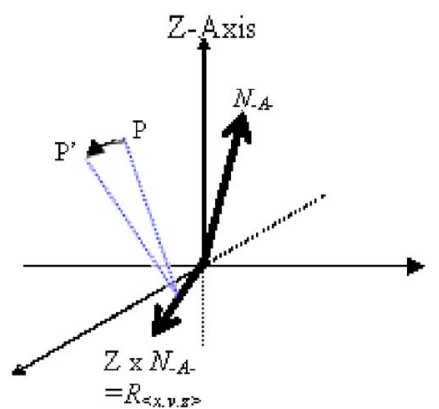

Fig. 5 Rotation axis and a rotation angle for leveling points to Datum -A- can be found by cross product between the Datum -A- plane normal vector and the $z$ axis.

viewed as orthogonal projections of the points onto the $x-y$ plane. Working in two dimensions, a "best-fit" line can be calculated for the set of Datum -B- points. Efficient algorithms are available for calculating, e.g., a linear leastsquares line through the data (e.g., Ref. 6). The "best-fit" line in the $x-y$ plane will also lie within the Datum -Bplane, based on the definition that Datum -A- and Datum -B- are orthogonal (and since the measured points were "leveled" to Datum -A-).

The "best-fit" line equation (in the $x-y$ plane) $y=m x+b$ can be used to find a vector that is parallel to the Datum -Bplane; then the cross-product of the vector with the Datum -A- normal vector will produce a normal vector for the Datum -B- plane. To find two points in the Datum -Bplane, choose two (arbitrary) $x$ values (e.g., $x=0$, and then $x=1)$, and use the line equation to calculate the points $(0, b, 0)$ and $(1, m+b, 0)$ on the line (and, therefore, within the Datum -B- plane). Then, a vector parallel to the Datum -B- plane can be found by subtracting the two points, which in this case would give the vector $\langle 1-0,(m+b)-b, 0\rangle$ $=\langle 1, m, 0\rangle$. The vector $\langle 1, m, 0\rangle$, where $m$ is the slope of the "best-fit" line, is a vector that is parallel to Datum -B-. Note that if the "best-fit" line algorithm returned a vertical line (i.e., with undefined slope $m$ ), then the vector $\langle 0,1,0\rangle$ would be parallel to the Datum -B- plane. But, because of the arbitrary designation that Datum -B- corresponds to the $x-z$ plane in the datum coordinate system, this case should not occur in practice, unless the measurement tool was rotated $90^{\circ}$ from its nominal position during precharacterization or post-mounting measurement.

The cross product of the vector $\langle 1, m, 0\rangle$, which is parallel to the Datum -B- plane, with the Datum -A- normal vector $N_{-A-}$ will produce a normal vector for the Datum -Bplane. This is because, by definition, Datum -A- and Datum -B- are orthogonal; and because the vector $\langle 1, m, 0\rangle$ is parallel to the Datum -B- plane. Since all of the Datum -Bpoints have been "leveled" to Datum -A-, the $z$-axis can be used in place of the Datum -A- normal, and the normal vector for the Datum -B- plane (unit length and pointing in the positive $y$ direction) is

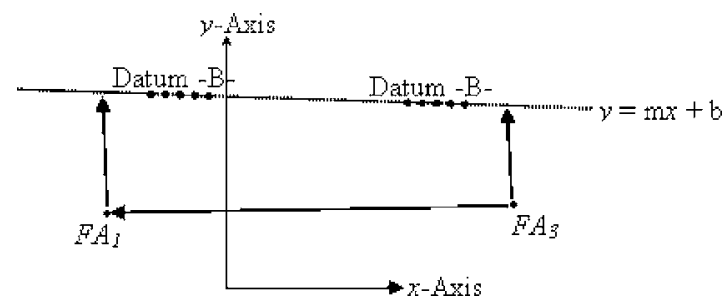

Fig. 6 "Plane transfer values" from the virtual point locations $F A_{1}$ and $F A_{3}$ on the Datum -A- plane, along a vector perpendicular to the vector from $F A_{3}$ to $F A_{1}$.

$N_{-B_{-}}=\langle\langle 0,0,1\rangle \times\langle 1, m, 0\rangle\rangle \cdot \frac{\operatorname{sign}[\langle\langle 0,0,1\rangle \times\langle 1, m, 0\rangle\rangle(2)]}{\|\langle\langle 0,0,1\rangle \times\langle 1, m, 0\rangle\rangle\|_{2}}$.

Using the fact that the point $(0, b, 0)$ lies within the Datum -B- plane, the corresponding plane equation for Datum -Bis

$N_{-B_{-}} \cdot\langle x, y, z\rangle=N_{-B_{-}} \cdot(0, b, 0)$,

where the right-hand side of the equation is the plane $d$ value $\left[d_{-B-}=N_{-B-} \cdot(0, b, 0)\right]$.

At this point, the Datum -B- plane equation has been established from tool precharacterization measurements. Now, the fixed relationship between the tool fiducials and the Datum -B- plane must be established so that Datum -Bcan be found only from measurements of the tool fiducials. The two "plane transfer values" for Datum -B- are distances from the virtual points $F A_{1}$ and $F A_{3}$ to the Datum -B- plane, along a vector in the Datum -A- plane that is normal to the vector from $F A_{3}$ to $F A_{1}$, i.e., the vector $\left\langle F A_{3}-F A_{1}\right\rangle$ (Fig. 6). The choice of $F A_{3}$ to $F A_{1}$ is made because of the location of these fiducials on the tool. Note $F A_{1}$ is near the datum origin, and $F A_{3}$ is out the positive $x$ axis of the measurement system. The direction of the vector $\left\langle F A_{3}-F A_{1}\right\rangle$ is generally along the $x$ axis, so a vector that is perpendicular to $\left\langle F A_{3}-F A_{1}\right\rangle$ will give a good path to the Datum -B- plane. This vector (unit length and pointing in the positive $y$ direction) is

$$
\begin{aligned}
F A_{-} B= & \left\langle N_{-A_{-}} \times\left\langle F A_{3}^{\prime}-F A_{1}^{\prime}\right\rangle\right\rangle \\
& \cdot \frac{\operatorname{sign}\left[\left\langle N_{-A_{-}} \times\left\langle F A_{3}^{\prime}-F A_{1}^{\prime}\right\rangle\right\rangle(2)\right]}{\left\|\left\langle N_{-A_{-}} \times\left\langle F A_{3}^{\prime}-F A_{1}^{\prime}\right\rangle\right\rangle\right\|_{2}} .
\end{aligned}
$$

Since the virtual points have been "leveled" to Datum -A-, this is equivalent to

$$
\begin{aligned}
F A_{-} B= & \left\langle\langle 0,0,1\rangle \times\left\langle F A_{3}^{\prime}-F A_{1}^{\prime}\right\rangle\right\rangle \\
& \cdot \frac{\operatorname{sign}\left\lfloor\left\langle\langle 0,0,1\rangle \times\left\langle F A_{3}^{\prime}-F A_{1}^{\prime}\right\rangle\right\rangle(2)\right\rfloor}{\left\|\left\langle\langle 0,0,1\rangle \times\left\langle F A_{3}^{\prime}-F A_{1}^{\prime}\right\rangle\right\rangle\right\|_{2}} .
\end{aligned}
$$

The "plane transfer values" for Datum -B-, per Eq. (9), are

$t_{4}=\frac{d_{-B-}-N_{-B-} \cdot F A_{1}^{\prime}}{N_{-B-} \cdot F A_{-} B}, \quad t_{5}=\frac{d_{-B-}-N_{-B-} \cdot F A_{3}^{\prime}}{N_{-B-} \cdot F A_{-} B}$.

The plane transfer values have a magnitude that is equal to the distance between each point and the Datum -B- plane, 
and a sign that depends on the direction of the $F A_{-} B$ vector. These values can be used to determine the Datum -Bplane from measurements of the tool fiducials during operational alignment or postmounting measurement.

The three virtual points $F A_{1}, F A_{2}$, and $F A_{3}$ in Datum -A-, with precharacterization measurements from the Datum -C- surface, can be used to determine the Datum -Cplane location. During tool precharacterization, several points are also measured on the edges of Datum -C-. With these measurements, the "plane transfer value" from the two virtual points $F A_{1}$ and $F A_{2}$ to the Datum -C-plane can be determined. To simplify the remaining calculations, the three "leveled" virtual points $F A_{1}^{\prime}, F A_{2}^{\prime}$, and $F A_{3}^{\prime}$, and all of the "leveled" points on Datum -C-, can be "skewed" to the Datum -B-plane. When this is done, the $y$ values for each of the measured points along Datum -C- become irrelevant. A rotation axis (and a rotation angle) for "skewing" points to Datum -B- can be found by cross-product (and dot product) between the Datum -B- plane normal vector and the $y$ axis:

$$
R_{\langle x, y\rangle}=\frac{\left\langle N_{-B-} \times\langle 0,1,0\rangle\right\rangle}{\left\|N_{-B_{-}} \times\langle 0,1,0\rangle\right\|_{2}}
$$

[The rotation axis for "skewing" to

$$
\text { -B-, a (unit-length) vector], }
$$

$\theta=\arccos \left[\frac{N_{-B-} \cdot\langle 0,1,0\rangle}{\left\|N_{-B-}\right\|_{2}}\right] \cdot\left\{-\operatorname{sign}\left(R_{\langle x, y, z\rangle}[3]\right)\right\}$

(The rotation angle, a scalar).

The rotation matrix corresponding to the rotation axis and rotation angle calculated by Eqs. (21a) and (21b), respectively, is

$\left[R_{\langle x, y\rangle}\right]=\left[\begin{array}{ccc}\cos \theta & \sin \theta & 0 \\ -\sin \theta & \cos \theta & 0 \\ 0 & 0 & 1\end{array}\right]$.

Note that for points that have been "leveled" to the Datum -A- plane [Eqs. (14)-(16)], the rotation axis for "skewing" to Datum -B- will always be the $z$ axis; however, the direction of rotation will be determined from the calculated rotation axis. This is shown in Eq. (22), where the direction of the rotation angle (a positive or negative angle) comes from whether the calculated rotation axis of Eq. (21a) points upward or downward. Points that have been "leveled" to the Datum -A- plane can be "skewed" to the Datum -B- plane by the 2-D rotation matrix

$P^{\prime}=\left\lfloor R_{\langle x, y\rangle}\right\rfloor \cdot P$,

where the third component ( $z$ component) of the original point $P$ is unchanged, since the rotation is about the $z$ axis. This rotation is applied to the three "leveled" virtual points $F A_{1}^{\prime}, F A_{2}^{\prime}$, and $F A_{3}^{\prime}$, and all of the "leveled" points on Datum -C-:

$F A_{i}^{\prime \prime}=\left[R_{\langle x, y\rangle}\right] \cdot F A_{i}^{\prime}, i=1 \ldots 3, C_{i}^{\prime \prime}=\left[R_{\langle x, y\rangle}\right] \cdot C_{i}^{\prime}, i=1 \ldots n_{-C_{-}}$.

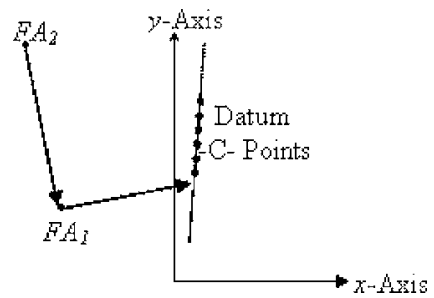

Fig. 7 "Plane transfer value" from the virtual point location $F A_{1}$ on the Datum -A- plane, along a vector perpendicular to the vector from $F A_{2}$ to $F A_{1}$.

For the purpose of determining die alignment, the three datum surfaces are assumed to be exact planes, i.e., perfectly flat and mutually orthogonal. Assuming that Datum -C- is perpendicular to both Datum -A- and Datum -B-, the normal vector for Datum -C- is just the cross product of

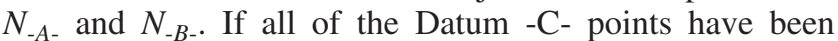
"leveled" to Datum -A- and "skewed" to Datum -B-, then $N_{-C-}$ is just the $x$ axis:

$N_{-C-}=\langle 1,0,0\rangle$.

To find the plane $d$ value for Datum -C-, a point within the Datum -C- plane must be determined. The Datum -Cpoints have been "leveled" to Datum -A- and "skewed" to Datum -B-. Taking the average of all the $x$ values of the "leveled" and "skewed" Datum -C-points will determine the point along the $x$ axis, where Datum -C-intersects the Datum -B-plane. Then, the plane $d$ value for Datum -Ccan be found using a dot product. Take the average "leveled" and "skewed" Datum -C- $x$ value, and calculate a dot product with the $x$ axis:

$d_{-C-}=\bar{x}_{C} \cdot N_{-C-}=\bar{x}_{-C-} \cdot\langle 1,0,0\rangle$.

When the plane equation for Datum -C- is known, the "plane transfer value" from the two virtual points $F A_{1}$ and $F A_{2}$ to the Datum -C-plane can be calculated. The "plane transfer value" is the distance from $F A_{1}$ to the Datum -Cplane along a vector perpendicular to $\left\langle F A_{2}-F A_{1}\right\rangle$ that also lies in Datum -A- Plane. The choice of $F A_{2}$ to $F A_{1}$ is made because of the location of these fiducials on the tool. Note $F A_{1}$ is near the datum origin, and $F A_{2}$ is out the positive $y$ axis of the measurement system. The direction of the vector $\left\langle F A_{2}-F A_{1}\right\rangle$ is generally along the $y$ axis, so a vector perpendicular to $\left\langle F A_{2}-F A_{1}\right\rangle$ will give a good path to the Datum -C-plane (Fig. 7). This vector (unit length and pointing in the positive $x$ direction) is

$$
\begin{aligned}
F A_{-} C= & \left\langle N_{-A_{-}} \times\left\langle F A_{2}^{\prime \prime}-F A_{1}^{\prime \prime}\right\rangle\right\rangle \\
& \cdot \frac{\operatorname{sign}\left\lfloor\left\langle N_{-A_{-}} \times\left\langle F A_{2}^{\prime \prime}-F A_{1}^{\prime \prime}\right\rangle\right\rangle(1)\right\rfloor}{\left\|\left\langle N_{-A_{-}} \times\left\langle F A_{2}^{\prime \prime}-F A_{1}^{\prime \prime}\right\rangle\right\rangle\right\|_{2}} .
\end{aligned}
$$

Since the virtual points have been "leveled" to Datum -Aand "skewed" to Datum -B-, this is equivalent to 


$$
\begin{aligned}
F A_{-} C= & \left\langle\langle 0,0,1\rangle \times\left\langle F A_{2}^{\prime \prime}-F A_{1}^{\prime \prime}\right\rangle\right\rangle \\
& \cdot \frac{\operatorname{sign}\left\lfloor\left\langle\langle 0,0,1\rangle \times\left\langle F A_{2}^{\prime \prime}-F A_{1}^{\prime \prime}\right\rangle\right\rangle(1)\right\rfloor}{\left\|\left\langle\langle 0,0,1\rangle \times\left\langle F A_{2}^{\prime \prime}-F A_{1}^{\prime \prime}\right\rangle\right\rangle\right\|_{2}} .
\end{aligned}
$$

The "plane transfer value" for Datum -C-, per Eq. (9), is

$t_{6}=\frac{d_{-C_{-}}-N_{-C_{-}} \cdot F A_{1}^{\prime \prime}}{N_{-C_{-}} \cdot F A_{-} C}$.

The plane transfer value has a magnitude that is equal to the distance between the point $F A_{1}$ and the Datum -Cplane along the $F A_{-} C$ vector, and a sign that depends on the direction of the $\bar{F} A_{-} C$ vector. This value will be used to determine the Datum -C- plane from measurements of the tool fiducials during post-mounting measurement.

\section{Operational Determination of Datum Surfaces}

In the context of active alignment or post-mounting measurement, the six "plane transfer values" can be used to determine the datum planes when only the tool fiducials are accessible to the $z$-scope measurement system. The fiducial plane equation is determined by equations (4) and (5), based on direct measurements with the $z$-scope. Three virtual points $F A_{1}, F A_{2}$, and $F A_{3}$ in the Datum -A- plane (Fig. 4) are calculated from the measured values of $F_{1}, F_{2}$, and $F_{3}$ and the fiducial plane equation by using the plane transfer values, and the normal vector to the fiducial plane [Eq. (11)]:

$$
\begin{gathered}
F A_{1}=F_{1}+t_{1} * N_{-F-}, \quad F A_{2}=F_{2}+t_{2} * N_{-F-}, \\
F A_{3}=F_{3}+t_{3} * N_{-F-} .
\end{gathered}
$$

The Datum -A- plane normal vector (unit length, and pointing upward) is then calculated from the three virtual points by Eqs. (12) and (13).

$$
\begin{aligned}
N_{-A_{-}=}= & \left\langle\left\langle F A_{2}-F A_{1}\right\rangle \times\left\langle F A_{3}-F A_{1}\right\rangle\right\rangle \\
& \cdot \frac{\operatorname{sign}\left[\left\langle\left\langle F A_{2}-F A_{1}\right\rangle \times\left\langle F A_{3}-F A_{1}\right\rangle\right\rangle(3)\right]}{\left\|\left\langle F A_{2}-F A_{1}\right\rangle \times\left\langle F A_{3}-F A_{1}\right\rangle\right\|_{2}} .
\end{aligned}
$$

Note $N_{-A_{-}}$is the Datum -A- plane normal vector, and the plane equation is

$N_{-A_{-}} \cdot\langle x, y, z\rangle=N_{-A_{-}} \cdot F A_{1}$,

where the right-hand side of the equation is the plane $d$ value $\left(d_{-A_{-}}=N_{-A_{-}} \cdot F A_{1}\right)$.

The two transfer values $t_{4}$ and $t_{5}$ can then be used to determine the Datum -B- plane. The fiducial plane equation is determined from measurements by Eqs. (4) and (5). Three virtual points $F A_{1}, F A_{2}$, and $F A_{3}$ in the Datum -Aplane (Fig. 4) are calculated from the measured values of $F_{1}, F_{2}$, and $F_{3}$ and the fiducial plane equation by using the plane transfer values $t_{1}, t_{2}$, and $t_{3}$ [Eq. (11)], and the normal vector to the fiducial plane [Eqs. (12) and (13)]. The vector perpendicular to the line from $F A_{3}$ to $F A_{1}$ (unit length and pointing in the positive $y$ direction) is also in the Datum -A- plane is calculated by Eq. (19a)

$$
\begin{aligned}
F A_{-} B= & \left\langle N_{-A_{-}} \times\left\langle F A_{3}-F A_{1}\right\rangle\right\rangle \\
& \cdot \frac{\operatorname{sign}\left\lfloor\left\langle N_{-A_{-}} \times\left\langle F A_{3}-F A_{1}\right\rangle\right\rangle(2)\right\rfloor}{\left\|\left\langle N_{-A_{-}} \times\left\langle F A_{3}-F A_{1}\right\rangle\right\rangle\right\|_{2}} .
\end{aligned}
$$

Then, two virtual points $F B_{1}$ and $F B_{2}$ in the Datum -Bplane are calculated using the Datum -B- "plane transfer values" of Eq. (20), by

$$
F B_{1}=F A_{1}+t_{4} * F A_{-} B, \quad F B_{2}=F A_{2}+t_{5} * F A_{-} B .
$$

The Datum -B- normal vector (unit length and pointing in the direction of the positive $y$ axis) is found by crossproduct of the vector between the two virtual points with the Datum -A- normal:

$$
\begin{aligned}
N_{-B_{-}=}= & \left\langle N_{-A_{-}} \times\left\langle F B_{2}-F B_{1}\right\rangle\right\rangle \\
& \cdot \frac{\operatorname{sign}\left[\left\langle N_{-A_{-}} \times\left\langle F B_{2}-F B_{1}\right\rangle\right\rangle(2)\right]}{\left\|\left\langle N_{-A_{-}} \times\left\langle F B_{2}-F B_{1}\right\rangle\right\rangle\right\|_{2}},
\end{aligned}
$$

and the Datum -B- plane equation is

$N_{-B-} \cdot\langle x, y, z\rangle=N_{-B-} \cdot F B_{1}$,

where the right-hand side of the equation is the plane $d$ value $\left(d_{-B-}=N_{-B-} \cdot F B_{1}\right)$.

The transfer value $t_{6}$ can then be used to determine the Datum -C- plane. The vector perpendicular to the line from the points $F A_{2}$ to $F A_{1}$ (unit length and pointing in the positive $x$ direction) that is also in the Datum -A-plane is calculated by equation (26a):

$$
\begin{aligned}
F A_{-} C= & \left\langle N_{-A_{-}} \times\left\langle F A_{2}-F A_{1}\right\rangle\right\rangle \\
& \cdot \frac{\operatorname{sign}\left\lfloor\left\langle N_{-A_{-}} \times\left\langle F A_{2}-F A_{1}\right\rangle\right\rangle(1)\right\rfloor}{\left\|\left\langle N_{-A_{-}} \times\left\langle F A_{2}-F A_{1}\right\rangle\right\rangle\right\|_{2}} .
\end{aligned}
$$

Then, the virtual point $F C_{1}$ in the Datum -C-plane is calculated using the Datum -C- "plane transfer value" of Eq. (27), by

$F C_{1}=F A_{1}+t_{6} * F A_{-} C$.

The Datum -C- normal vector (unit length and pointing in the direction of the positive $x$ axis) is found by cross product of the vector between the two other datum normal vectors:

$N_{-C_{-}}=\left\langle N_{-A_{-}} \times N_{-B-}\right\rangle \cdot \frac{\operatorname{sign}\left[\left\langle N_{-A_{-}} \times N_{-B_{-}}\right\rangle(1)\right]}{\left\|\left\langle N_{-A_{-}} \times N_{-B-}\right\rangle\right\|_{2}}$,

and the Datum -C-plane equation is

$N_{-C-} \cdot\langle x, y, z\rangle=N_{-C-} \cdot F C_{1}$,

where the right-hand side of the equation is the plane $d$ value $\left(d_{-C_{-}}=N_{-C_{-}} \cdot F C_{1}\right)$.

The three datum planes are mutually orthogonal, and the point of intersection of all three planes represents the origin point of the datum coordinate system. The three plane equations form a system of three equations in three unknowns, where the solution of the system of equations is the origin point:

$N_{-A_{-}}(1) \cdot x+N_{-A_{-}}(2) \cdot y+N_{-A-}(3) \cdot z=d_{-A_{-}}$, 
$N_{-B-}(1) \cdot x+N_{-B-}(2) \cdot y+N_{-B-}(3) \cdot z=d_{-B-}$,

$N_{-C_{-}}(1) \cdot x+N_{-C_{-}}(2) \cdot y+N_{-C_{-}}(3) \cdot z=d_{-C_{-}} \cdot$

The system of equations can be solved, for example, by Gaussian elimination with pivot scaling and back substitution (e.g., Ref. $6^{6}$ ). However, since the datum planes are mutually orthogonal, and are roughly aligned with the measurement system coordinate planes, the numerical system should be stable, and an algebraic solution (e.g., based on Cramer's rule) can be used without fear of numerical quirkiness. The advantage of using an algebraic solution is that the code is simpler (and probably more efficient) than implementing a Gaussian elimination scheme. The following algebraic solution to the linear system in Eq. (34) is from Cramer's rule, with some factoring to streamline the calculations.

denom $=\left[N_{-A_{-}}(2) \cdot N_{-B_{-}}(3)-N_{-A_{-}}(3) \cdot N_{-B_{-}}(2)\right] \cdot N_{-C_{-}}(1)+\left[N_{-A_{-}}(3) \cdot N_{-C_{-}}(2)-N_{-A_{-}}(2) \cdot N_{-C_{-}}(3)\right] \cdot N_{-B_{-}-}(1)+\left[N_{-B_{-}}(2) \cdot N_{-C_{-}}(3)-N_{-B_{-}-}(3) \cdot N_{-C_{-}}(2)\right] \cdot N_{-A_{-}-}(1)$,
Datum origin $(1)=\frac{\left[N_{-B_{-}}(2) \cdot N_{-C_{-}}(3)-N_{-B_{-}-}(3) \cdot N_{-C_{-}}(2)\right] \cdot d_{-A_{-}}+\left[N_{-A_{-}}(3) \cdot N_{-C_{-}}(2)-N_{-A_{-}-}(2) \cdot N_{-C_{-}}(3)\right] \cdot d_{-B_{-}}+\left[N_{-A_{-}}(2) \cdot N_{-B_{-}}(3)-N_{-A_{-}}(3) \cdot N_{-B_{-}}(2)\right] \cdot d_{-C_{-}}}{\operatorname{denom}}$

Datum origin $(2)=\frac{\left[N_{-B_{-}}(3) \cdot N_{-C_{-}}(1)-N_{-B_{-}}(1) \cdot N_{-C_{-}}(3)\right] \cdot d_{-A_{-}}+\left[N_{-A_{-}}(1) \cdot N_{-C_{-}}(3)-N_{-A_{-}}(3) \cdot N_{-C_{-}}(1)\right] \cdot d_{-B_{-}}+\left[N_{-A_{-}}(3) \cdot N_{-B_{-}}(1)-N_{-A_{-}}(1) \cdot N_{-B_{-}}(3)\right] \cdot d_{-C_{-}}}{\operatorname{denom}}$

Datum origin (3) $=\frac{\left[N_{-B_{-}}(1) \cdot N_{-C_{-}}(2)-N_{-B_{-}}(2) \cdot N_{-C_{-}}(1)\right] \cdot d_{-A_{-}}+\left[N_{-A_{-}}(2) \cdot N_{-C_{-}}(1)-N_{-A_{-}}(1) \cdot N_{-C_{-}}(2)\right] \cdot d_{-B_{-}}+\left[N_{-A_{-}}(1) \cdot N_{-B_{-}}(2)-N_{-A_{-}}(2) \cdot N_{-B_{-}}(1)\right] \cdot d_{-C_{-}}}{\operatorname{denom}}$

A denominator close to zero would indicate that the linear system is close to being singular. This can be checked after just 14 operations (which is probably quicker than a Gaussian elimination scheme). However, if all the measurements are valid, the singular case should not occur.

At this point, all of the algorithms required for determining the datum planes from precharacterization measurements of the tool fiducials and datum surfaces have been presented. During postmounting measurement, the datum plane equations are calculated from precharacterization data (or the six "plane transfer values" which can be calculated previously) and measurements of the tool fiducials. Tool fiducial measurements are made in the measurement coordinate system. The three datum plane equations thus show where the datum planes are within the measurement coordinate system; "translation," "level," and "skew" transformations from the measurement coordinate system to the datum coordinate system are based on the plane equations. To calculate die alignment according to a print specification, the appropriate die feature is measured, and the location is "translated," "leveled," and "skewed" to the datum coordinate system. The transformed location can be compared directly to the print specification for determining whether die alignment meets the specification. Source code that implements these algorithms is available online. ${ }^{8}$

\section{Notes About Accuracy, Precision}

The algorithms presented in this paper were successfully implemented for ultraprecision, active manufacturing alignment, and postalignment measurement of IR imaging systems. The accuracy of the final alignment depends on the measurement uncertainty of the alignment system (and to some degree on the temperature conditions of the manufacturing environment). The algorithms do not (under normal circumstances) cause small measurement errors to blow up and give erroneous results. Since repeated measurements of the datum surfaces are used, standard statistical techniques can be used to estimate measurement error. The magnitude of alignment uncertainty can be calculated by allowing the measurement uncertainty to propagate through the algorithm calculations. Volumetric calibration of the measurement system is required, and for high-precision alignment, the use of nonlinear error correction for all three stage axes is essential. Using high-quality optics and sufficiently corrected stages, measurement guard bands for alignment errors are significantly better than manufacturing tolerances for FPA mounting platforms.

\section{References}

1. L. Shin and G. S. Winters, "NICMOS flight focal plane assembly," in Astronomical Telescopes and Instruments II, P. Y. Bely and J. B. Breckinridge, Eds., Proc. SPIE 1945, 371-382 (1993).

2. K. E. Yokoyama, H. Miller, Jr., T. Hedman, S. Thordarson, M Figueroa, J. Shepanski, P. J. Jarecke, and S. Lai, "NGST longwave hyperspectral imaging spectrometer system characterization and calibration," in Imaging Spectrometry IX, S. S. Shen, and P. E. Lewis, Eds., Proc. SPIE 5159, 262-274 (2003).

3. J. A. Stone, M. Amer, B. Faust, and J. Zimmerman, "Uncertainties in small-angle measurement systems used to calibrate angle artifacts," $J$. Res. Natl. Inst. Stand. Technol. 109(3), 319-333 (2004).

4. P. Lehmann "Optical versus tactile geometry measurement: alternatives or counterparts," in Optical Measurement Systems for Industrial Inspection III, W. Osten, M. Kujawinska, and C. Creath, Eds., Proc. SPIE 5144, 183-196 (2003).

5. H. Spaeth, Mathematical Algorithms for Linear Regression, Elsevier Academic Press, San Diego, CA (1991).

6. W. H. Press, S. A. Teukolsky, W. T. Vetterling, and B. P. Flannery, Numerical Recipes in C, The Art of Scientific Computing, 2nd ed., Cambridge University Press, New York (1992).

7. G. Strang Calculus, Wellesley-Cambridge Press, Wellesley, MA (1991).

8. Source code (in c) that implements these algorithms is available at the StatLib archive, maintained by Pantelis Vlachos at the Department of Statistics, Carnegie Mellon University (http:// lib.stat.cmu.edu/). 
Gary B. Hughes is currently a senior systems engineer with FLIR Systems Inc., Indigo Operations, in Goleta, California. He works in manufacturing and calibration of uncooled microbolometer-array cameras that are used in night vision systems. He received his BA degree in mathematics from Northwestern University, Evanston, Illi- nois, in 1986, his MA degree in applied mathematics from the University of California, Santa Barbara, in 1992, and his PhD degree in earth and environmental science from the University of Pennsylvania, Philadelphia, in 1999. 